\title{
ON THE FACIAL STRUCTURE OF A CONVEX BODY
}

\author{
J. B. COLLIER
}

\begin{abstract}
The family formed by taking the relative interior of each face of a $d$-dimensional convex body $C$ is a partition of $C$. It is shown here that the subfamily consisting of all the $(d-2)$-dimensional sets has a quotient topology which is paracompact and this is used to prove a property of the set of extreme points when $d=3$.
\end{abstract}

A convex body in $E^{d}$ is any closed bounded convex subset $C$ with nonempty interior. A flat is any translate of a subspace. A face of $C$ is a convex subset $F$ which is the intersection of $C$ with a flat such that $C \backslash F$ is also convex. Thus the extreme points of $C$ are exactly the 0 -dimensional faces of $C$. The set of extreme points of $C$ will be denoted by ext $C$ and for any set $A$ the boundary of $A$ and the closure of $A$ will be denoted by bd $A$ and $\operatorname{cl} A$, respectively.

It is easily seen that if $C$ is 2-dimensional, then ext $C$ is a closed set. This need not be true in higher dimensions. In fact, there is a convex body $C$ in $E^{3}$ for which ext $C$ and bd $C \backslash \operatorname{ext} C$ are both dense in bd $C$ (see [4, p. 104]). However, we can show the following relationship between ext $C$ and its closure:

THEOREM 1. If $C$ is a convex body in $E^{3}$, then each component of $\operatorname{cl}(\operatorname{ext} C) \backslash \operatorname{ext} C$ is a subset of a 1-dimensional face of $C$.

Klee raised the question [4] of characterizing the family $\chi$ of all subsets $K$ of the 2-sphere, $S^{2}$, for which there is a convex body $C$ in $E^{3}$ and a homeomorphism $h$ of $S^{2}$ onto bd $C$ such that $h[K]=\operatorname{ext} C$. In [1] it was shown that if $\mathrm{cl} K$ is 0 -dimensional, then $K \in \chi$ if and only if $K$ is a $G_{\delta}$ set. Theorem 1 indicates that this result cannot be substantially generalized. For example, if $K$ is a countable subset of $S^{2}$, but $\operatorname{cl}[K] \backslash K$ is a circle, then $K \notin \chi$.

Our proof of Theorem 1 depends on an interesting property of the 1dimensional faces of a convex body in $E^{3}$. More generally, we consider for any convex body $C$ in $E^{d}, d \geqslant 3$, the family $\mathfrak{L}(C)=\{\operatorname{ri} F \mid F$ is a $(d-2)$ dimensional face of $C$, where ri $F$ denotes the relative interior of $F$. Recall that the relative interior of $F$ is the interior of $F$ relative to the smallest flat containing it. It is easily seen that $\mathscr{L}(C)$ is a family of pairwise disjoint subsets of bd $C$ and, consequently, the usual quotient topology may be associated with

Received by the editors July 1, 1975.

AMS (MOS) subject classifications (1970). Primary 52A15, 52A20.

(c) American Mathematical Society 1977 
it. In general, this topology fails to be metrizable; however, we can show the following:

THEOREM 2. For any convex body $C$ in $E^{d}, \mathcal{L}(C)$ is paracompact.

This may be compared to the easily observed fact that the quotient topology for the family $\{$ ri $F \mid F$ is a $(d-1)$-dimensional face of $C\}$ is countable and discrete.

We assume in the remainder of the paper that $C$ is a convex body in $E^{d}, d \geqslant 3, X=\cup\{A \mid A \in \mathcal{L}(C)\}$, and $\iota: X \rightarrow \mathcal{L}(C)$ is defined by $x \in \iota(x)$. Thus the quotient topology on $\varrho(C)$ is the largest topology which makes $\iota$ continuous. Let $Y$ be the set of all points in bd $C$ which do not lie in the relative interior of some $(d-1)$-dimensional face of $C$. Clearly $Y$ is a closed set containing $X$. Moreover, $X$ is an $F_{\sigma}$ set since $X=\cup_{n=1}^{\infty} K_{n}$ where $K_{n}$ is the set of all points which are the center of some closed $(d-2)$-dimensional ball of radius $1 / n$ contained in $Y$. Any limit point of $K_{n}$ also has this property; hence $K_{n}$ is closed.

For any $S \subseteq E^{d}$ and $\varepsilon>0$, let $N(S, \varepsilon)$ be the open set of all points that lie within $\varepsilon$ of $S$.

Lemma 1. For each $A \in \mathfrak{L}(C)$ and $\varepsilon>0$, there is a closed neighborhood of $A$ in $\mathcal{L}(C)$ whose members are contained in $N(A, \varepsilon)$.

Proof. An open subset $U$ of bd $C$ will be called $L$-open if for each $B \in \mathfrak{L}(C), U \cap B \neq \varnothing$ implies that $B \subseteq U$. In this case $\mathscr{U}=\{B \in \mathfrak{L}(C) \mid$ $U \cap B \neq \varnothing\}$ is open in $\mathscr{E}(C)$. Let $K=$ bd $C \backslash N(A, \varepsilon)$ and $a_{1} \in A$. Since $K$ is compact, it suffices to show that for each $x \in K, a_{1}$ and $x$ have disjoint $L$ open neighborhoods.

Let $a_{2} \in K$ and let $F$ be the smallest face of $C$ containing both $a_{1}$ and $a_{2}$. Since $a_{1}$ is in the relative interior of a $(d-2)$-dimensional face, either $F=C$ or $F$ has dimension $d-1$. Let $M$ be the subspace of codimension 1 which is perpendicular to the line through $a_{1}$ and $a_{2}$ and let $\pi$ be the orthogonal projection of $E^{d}$ onto $M$. Thus $\pi\left(a_{1}\right)=\pi\left(a_{2}\right)$. Choose some point $b$ on the line $M^{\perp}$ other than the origin and for $m \in \pi[C]$ define the functions $f_{1}(m)$ $=\inf \{r \in \mathbf{R} \mid m+r b \in C\}$ and $f_{2}(m)=\sup \{r \in \mathbf{R} \mid m+r \dot{b} \in C\}$. We may assume $a_{i}$ is in the graph of $f_{i}$. Clearly $f_{1}$ is convex and $f_{2}$ is concave.

Let $U_{i}=\left\{\left(x, f_{i}(x)\right) \mid x \in \operatorname{ri} \pi[C]\right\}$; then $U_{1}$ and $U_{2}$ are disjoint $L$-open sets. If $F=C$, then $a_{i} \in U_{i}$ and we are done. If $F \neq C$, let $V_{i}=\left\{\left(x, f_{i}(x)\right)\right\}$ $x \in \operatorname{ri} \pi[F]\}$. Choose a point $q \in \operatorname{ri} F$, let $W_{i}$ be the convex hull of $V_{i} \cup\{q\}$, and let $V_{i}^{\prime}=V_{i} \cup$ ri $W_{i}$. Then $U_{i}^{\prime}=U_{i} \cup V_{i}^{\prime}$ is an $L$-open set containing $a_{i}$ and $U_{1}^{\prime}, U_{2}^{\prime}$ are disjoint.

Proof of TheOrem 2. Lemma 1 implies that $\ell(C)$ is Hausdorff and the second countability of $E^{d}$ implies $\mathcal{L}(C)$ is Lindelöf. Therefore, by a theorem of Morita [2, p. 174], it is sufficient to show $\mathcal{L}(C)$ regular in order to show that it is paracompact. Let $\mathscr{K}$ be any closed subfamily of $\mathscr{L}(C)$ and $A \in \mathcal{L}(C) \backslash \mathfrak{K}$. We exhibit a closed neighborhood of $\mathscr{K}$ not containing $A$.

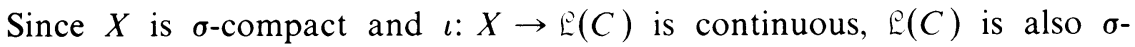


compact. Hence $\mathscr{K}$ is the union of some sequence $\mathscr{K}_{1} \subseteq K_{2} \subseteq \cdots$ of compact subfamilies of $\mathcal{L}(C)$. Let $K_{n}=\bigcup\left\{B \mid B \in \mathcal{K}_{n}\right\}$. It follows from Lemma 1 and the compactness of $\mathscr{K}_{n}$ that $\mathscr{K}_{n}$ has a closed neighborhood $\mathscr{W}_{n}$ contained in $N\left(K_{n}, 1 / n\right)$ such that $A \notin \mho_{n}$. Let $\mho_{n}=\cup_{n=1}^{\infty} \mho_{n}, W_{n}=\cup\left\{B \mid B \in \mho_{n}\right\}, W$ $=\cup_{n=1}^{\infty} W_{n}$, and $K=\cup_{n=1}^{\infty} K_{n}$. Clearly, and $K_{n} \subseteq W_{n}$ for each $n$.

Since $\mho$ is closed if and only if $W$ is closed relative to $X$, suppose that there is an $x \in(X \cap \operatorname{cl} W) \backslash W$. Because $W_{n}$ is closed relative to $X$ for each $n, x$ must be in the closure of $\cup_{n=k}^{\infty} W_{n}$ for each $k$. Hence $x \in N(K, 1 / k)$ for each $k$. Since $K$ is closed relative to $X, x \in K$. This is a contradiction since $K \subseteq W$ and the theorem follows.

REMARK. A straightforward modification of the above proof shows that, in fact, any subfamily of $\mathfrak{L}(C)$ is paracompact.

Proof of Theorem 1 . Let $C$ be a convex body in $E^{3}$ and $D$ $=\operatorname{cl}(\operatorname{ext} C) \backslash \operatorname{ext} C$; then ext $C$ and $X$ form a partition of the closed set $Y$ and therefore $D \subseteq X$. Let $\mathscr{K}=\{A \in \mathfrak{L}(C) \mid A \cap D \neq \varnothing\}$ and $K=\cup\{A \mid A$ $\in \mathscr{K}\}$. Since $\iota: X \rightarrow \mathfrak{L}(C)$ is continuous, components of $D$ are mapped into components of $\mathcal{K}_{0}$ In the remainder of the proof we show that $\mathcal{K}$ must be totally disconnected and this implies Theorem 1 .

Since $X$ is an $F_{\sigma}, D$ is an $F_{\sigma}$. Let $D$ be the union of compact subsets $D_{1}, D_{2}, \ldots$ and let $\mathscr{D}_{n}=\left\{A \in \mathcal{L}(C) \mid A \cap D_{n} \neq \varnothing\right\}$. The continuity of $\iota$ implies that $\mathscr{D}_{n}$ is compact and therefore closed since $\mathcal{L}(C)$ is Hausdorff. Thus each set $D_{n}^{\prime}=\bigcup\left\{A \mid A \in \mathscr{D}_{n}\right\}$ is closed relative to $X$ and must also be an $F_{\sigma}$. It follows that $K=\cup_{n=1}^{\infty} D_{n}^{\prime}$ is an $F_{\sigma}$ set. Let $K$ be the union of the sequence $V_{1} \subseteq V_{2} \subseteq \cdots$ of compact sets.

For each rational number $r$ and each integer $i, 1 \leqslant i \leqslant 3$, the set $\left\{\left(x_{1}, x_{2}, x_{3}\right) \in E^{3} \mid x_{i}=r\right\}$ is a plane in $E^{3}$. Let $H_{1}, H_{2}, \ldots$ be an enumeration of these planes. If $A$ is any member of $\mathscr{K}$, there is an integer $N$ such that for each $n \geqslant N, A \cap V_{n}$ contains an open line segment. Thus for some $n \geqslant N$ there is an $H_{n}$ which intersects $A \cap V_{n}$ in a single point. Let $K_{n}$ be the union of all singleton sets of the form $A \cap V_{n} \cap H_{n}$ where $A \in \mathcal{K}$. If $x \in$ $\left(V_{n} \cap H_{n}\right) \backslash K_{n}$, then $x \in A$ for some $A \in \mathcal{K}$ but $A \cap V_{n} \cap H_{n}$ contains more than one point. In this case $A \subseteq H_{n}$. If $K_{n} \neq \varnothing$, then $H_{n} \cap$ ri $C \neq \varnothing$ and $A$ is open relative to bd $C \cap H_{n}$, which implies that $K_{n}$ is a closed set. Let $\mathscr{K}_{n}=\left\{A \in \mathscr{K} \mid A \cap K_{n} \neq \varnothing\right\}$; then $\mathscr{K}=\cup_{n=1}^{\infty} \mathcal{K}_{n}$. Moreover, since $\iota / K_{n}$ is one-to-one, $K_{n}$ is compact, and $\mathscr{L}(C)$ is Hausdorff, it follows that $K_{n}$ is homeomorphic to $K_{n}$.

For each $x \in X$, let $f(x)$ be the smallest face of $C$ containing $x$. Clearly, $f(x)=\operatorname{cl} \iota(x)$. If $\left\{a_{n}\right\}$ is a sequence in $X$, then $\varlimsup$ im $f\left(a_{n}\right)=\left\{x \mid f\left(a_{n}\right)\right.$ frequently intersects each neighborhood of $x\}$ and $\underline{\lim } f\left(a_{n}\right)=\left\{x \mid f\left(a_{n}\right)\right.$ eventually intersects each neighborhood of $x\}$. The function $f$ is called upper semicontinuous [resp., lower semicontinuous] at $a \in X$ if for each sequence $\left\{a_{n}\right\}$ in $X$ converging to $a, \overline{\lim } f\left(a_{n}\right) \subseteq f(a)\left[\operatorname{resp} ., \underline{\lim } f\left(a_{n}\right) \supseteq f(a)\right]$. A slight modification of a theorem of Klee and Martin [5, p. 6] shows that $f$ is upper semicontinuous at each point of $X$. 
Suppose $K_{n}$ contains an open line segment $S$. A theorem of Fort [3, p. 287] implies that $f / S$ is lower semicontinuous, as well as upper semicontinuous, at some point $s \in S$. It follows from this that $X$ is a neighborhood of $\iota(s)=A$ in bd $C$. Since this contradicts the assumption that $\operatorname{cl}(\operatorname{ext} C) \cap A \neq \varnothing, K_{n}$ can contain no open line segment. Hence $K_{n}$ is 0-dimensional.

Suppose $A_{1}$ and $A_{2}$ are distinct members of $K_{\text {. For }} i=1,2$, we construct sequences $\mathcal{W}_{0}^{i} \subseteq \mathcal{W}_{1}^{i} \subseteq \cdots$ of closed neighborhoods of $A_{i}$ in $\mathfrak{L}(C)$ such that $\mathscr{K}_{n}$ lies in the interior of $\mathcal{W}_{n}^{1} \cup \mathcal{W}_{n}^{2}$ but $\mathcal{W}_{n}^{1} \cap \mathcal{W}_{n}^{2}=\varnothing$. Recall that $\mathfrak{L}(C)$ is normal since it is paracompact. Let $\mathscr{w}_{0}^{1}$ and $\mathscr{\omega}_{0}^{2}$ be any disjoint closed neighborhoods of $A_{1}$ and $A_{2}$, respectively. Assume that $\mho_{n-1}^{1}$ and $\mho_{n-1}^{2}$ have been constructed for some $n \geqslant 1$. Let $\mathcal{K}_{n}^{i}=\mathcal{K}_{n} \cap \mathscr{W}_{n-1}^{i}$. Since $\mathcal{K}_{n}$ is homeomorphic to the compact, 0-dimensional set $K_{n}$ and $\mathcal{K}_{n}^{1}$ and $\mathcal{K}_{n}^{2}$ are disjoint closed subfamilies of $\mathcal{K}_{n}$, there exist disjoint closed subfamilies $\mathscr{P}_{n}^{i} \supseteq \mathcal{K}_{n}^{i}$ such that $\mathcal{K}_{n}=\mathscr{P}_{n}^{1} \cup \mathscr{P}_{n}^{2}$. Let $\mathscr{W}_{n}^{1}$ and $\mathscr{W}_{n}^{2}$ be disjoint closed neighborhoods of $\mathcal{Q}_{n-1}^{1} \cup \mathcal{P}_{n}^{1}$ and $\mathcal{Q S}_{n-1}^{2} \cup \mathcal{P}_{n}^{2}$, respectively. Let $\mathscr{W}_{i}$ be the interior of $\cup_{n=1}^{\infty} \mathscr{W}_{n}^{i}$; then $\mathscr{W}_{1}$ and $\mathscr{W}_{2}$ are disjoint neighborhoods of $A_{1}$ and $A_{2}$, respectively, and $\mathscr{K} \subseteq \mathscr{W}_{1} \cup \mathscr{W}_{2}$. Therefore $A_{1}$ and $A_{2}$ belong to different components of $K_{\text {. }}$ Since $A_{1}$ and $A_{2}$ were arbitrary members of $\mathscr{K}, \mathcal{K}$ is totally disconnected.

\section{REFERENCES}

1. J. B. Collier, On the set of extreme points of a convex body, Proc. Amer. Math. Soc. 47 (1975), 184-186. MR 50 \#117.

2. J. Dugundji, Topology, Allyn and Bacon, Boston, Mass., 1966. MR 33 \# 1824.

3. M. K. Fort, Jr., Category theorems, Fund. Math. 42 (1955), 276-288. MR 17, 1115.

4. V. L. Klee, Extremal structure of convex sets. II, Math. Z. 69 (1958), 90-104. MR 19, 1065.

5. V. L. Klee and M. Martin, Semicontinuity of the face-function of a convex set, Comment. Math. Helv. 46 (1971), 1-12. MR 43 \# 7903.

Department of Mathematics, University of Southern California, Los Angeles, CaliFORNIA 90007 